\title{
Extreme Consistency: Overcoming Annotation Scarcity and Domain Shifts
}

\author{
Gaurav Fotedar*, Nima Tajbakhsh*, Shilpa Ananth, and Xiaowei Ding \\ VoxelCloud Inc. \\ \{gauravfo, ntajbakhsh, shilpa, xding\}@voxelcloud.io
}

\begin{abstract}
Supervised learning has proved effective for medical image analysis. However, it can utilize only the small labeled portion of data; it fails to leverage the large amounts of unlabeled data that is often available in medical image datasets. Supervised models are further handicapped by domain shifts, when the labeled dataset, despite being large enough, fails to cover different protocols or ethnicities. In this paper, we introduce extreme consistency, which overcomes the above limitations, by maximally leveraging unlabeled data from the same or a different domain in a teacherstudent semi-supervised paradigm. Extreme consistency is the process of sending an extreme transformation of a given image to the student network and then constraining its prediction to be consistent with the teacher network's prediction for the untransformed image. The extreme nature of our consistency loss distinguishes our method from related works that yield suboptimal performance by exercising only mild prediction consistency. Our method is 1) auto-didactic, as it requires no extra expert annotations; 2) versatile, as it handles both domain shift and limited annotation problems; 3) generic, as it is readily applicable to classification, segmentation, and detection tasks; and 4) simple to implement, as it requires no adversarial training. We evaluate our method for the tasks of lesion and retinal vessel segmentation in skin and fundus images. Our experiments demonstrate a significant performance gain over both modern supervised networks and recent semi-supervised models. This performance is attributed to the strong regularization enforced by extreme consistency, which enables the student network to learn how to handle extreme variants of both labeled and unlabeled images. This enhances the network's ability to tackle the inevitable same- and cross-domain data variability during inference.
\end{abstract}

Keywords: Limited annotation, domain shift, semi-supervised learning

\section{Introduction}

Deep learning has proved effective in automating the diagnosis and quantification of various diseases and conditions. However, the vast majority of such models have limited clinical value, because they are trained and evaluated on labeled datasets that lack adequate annotation or show poor data diversity. The former problem is mainly caused by limited annotation budgets, while the latter, which 
causes the domain shift problem, arises when a dataset has poor coverage of ethnicities, imaging protocols, and scanner devices. In practice, securing a large, diverse labeled dataset is hardly feasible if not impossible; therefore, it is critical to devise learning strategies that increase model robustness against such dataset limitations through the effective utilization of unlabeled data.

To mitigate both limited annotation and domain shift problems, we propose a Semi-Supervised Learning (SSL) method, called Extreme Consistency. The main idea behind extreme consistency is to regularize the model by forcing predictions for the original data and its extreme transformations to be consistent. This idea is fundamentally different from data augmentation (Table 2(c)), because the latter can be applied only to labeled data and, further, lacks explicit consistency constraints. We realize our method through a teacher-student paradigm, where we feed an extreme variant of a given image to the student network and constrain its prediction to be consistent with the teacher's prediction for the original image. Importantly, extreme consistency relies on a diverse set of intensity-based, geometric, and image mixing transformations, which are blended based on a prior probability. If the combined transformation consists of only intensity manipulations, consistency loss reduces to an invariance constraint; that is, the predictions for the original and transformed image must be identical. However, inclusion of geometric and mixing augmentation will elevate this to an equivariance constraint; i.e., predictions must exhibit the same geometric transformations applied to the original images. Owing to the stochastic nature of diverse transformations, extreme consistency effectively spawns numerous invariance and equivariance constraints, providing strong regularization and thus higher-performing models. Critically, we exclusively apply diverse transformations to the inputs of the student network; thus, the teacher will receive "easy" examples that have only mild augmentation, which facilitates a healthy reference prediction for the consistency loss. Our experimental results on three public datasets demonstrate that the exclusive and diverse transformations make critical contributions to the success of our method, enabling a new level of performance that surpasses recent semi-supervised models $[18,6,21,8]$, as well as supervised models based on U-Net and UNet++ [29].

Extreme consistency offers the following advantages: First, our method is auto-didactic, because extreme consistency uses the teacher's predictions on unlabeled data, requiring no additional expert annotations. Second, our method is versatile, handling both domain shift and limited annotation problems. This is because extreme consistency exposes the model to extreme variants of labeled and unlabeled data, which enhances the capability to cope with future sameand cross-domain data disparity. Third, our method is generic because extreme consistency can be applied to classification, segmentation, and detection tasks by subjecting logits, masks, and saliency maps to the extreme consistency loss, respectively. Note that saliency or center maps are used in recent object detectors such as CenterNet [9]. Fourth, our method is simple to implement, requiring neither adversarial training nor any changes in the architecture of the base model.

Our contributions include 1) a novel semi-supervised approach based on extreme consistency, 2) a cross-dataset image mixing transformation to expand 
the unlabeled data for stronger consistency regularization, 3) a comprehensive ablation study on the individual components of our method, 4) extensive evaluation using three public datasets for the problems of limited annotations and domain shifts, and 5) demonstrated gains over several semi-supervised baselines as well as supervised baselines trained only with labeled data.

\section{Related Work}

In this section, we briefly review the semi-supervised learning and unsupervised domain adaptation methods that are most relevant to our work, and refer the readers to [24] for a comprehensive review of these methodologies.

Early SSL methods were based on adversarial zero-sum games, which, at their equilibrium, generate similar feature embedding [22,1] or similar predictions $[28,19]$ for labeled and unlabeled data. Recent SSL methods have, however, strived for simplicity and practicality. One of the popular recent themes is to train the model so it generates consistent results for a given image undergoing certain transformations. The consistency loss in $[6,26]$ encourages invariance against only noise perturbations, while the consistency loss in $[2,18]$ encourages equivariance against geometric transformations. Finally, the consistency loss in [21] is similar to $[2,18]$, but benefits from a teacher-student paradigm. Our method is also based on consistency loss; however, it is not limited to certain geometric transformations (flip and rotation in [18]) or certain types of additive or multiplicative noise perturbations (Gaussian noise in $[6,26]$ ). This is because specific transformations may only be effective for a limited range of applications. For instance, consistency under perturbation may not be as effective in ultrasound images or optical coherence tomography scans that have inherent speckle noise or low signal-tonoise ratio. A concurrent work similar to ours is the arXiv submission [23], which considers only image classification and tackles the limited annotation problem in the context of natural images. By contrast, our paper tackles image segmentation in medical images, benefits from image mixing augmentation, and targets both domain shift and scare annotation problems.

The domain adaptation methods fall into two major categories. The first consists of methods that convert labeled source images such that they take the appearance and style of the target domain images [15,5], whereas the second converts the target domain images into the source domain images $[4,13]$. Despite the differences, methodologies in both categories heavily rely on different variants of adversarial networks. By contrast, our approach is easy to train, requiring no sophisticated adversarial training.

\section{Method}

Our semi-supervised learning method follows the teacher-student paradigm. Specifically, our architecture consists of a student network $N_{s}$, whose weights are updated through backpropagation, and a teacher network $N_{t}$ whose weights simply track the weights of the student network through an exponential moving 
average filter. During training, the student network has two learning objectives: 1) minimizing the target supervised loss for the labeled data, 2) minimizing an unsupervised consistency loss by generating predictions that are consistent with the reference predictions generated by the teacher network. The novel aspect of our method is the notion of extreme consistency, which maximally utilizes unlabeled data during training.

Extreme consistency: Extreme consistency is the process of feeding an extreme variant of a given image to the student network and constraining its prediction to be consistent with the teacher's prediction for the original image. The extreme nature of our consistency loss distinguishes our method from similar works (e.g., $[18,26,6])$ that exercise prediction consistency under a limited set of transformations. We take the prediction consistency to extremes through a diverse set of transformations, which effectively spawns numerous invariance and equivariance constraints on the student network. Invariance constraints are imposed when the two networks receive variants of the same input that differ only in intensity values with no change in the spatial layout; otherwise, the applied transformation leads to an equivariance constraint; that is, the resulting predictions from the two networks must reflect the same geometric differences as do the original and transformed images. We hypothesize that extreme consistency provides stronger regularization, thereby enabling a higher-performing student network.

Diverse transformations: We generate diverse variants of an input image through an extreme image transformation module $T_{e}$. For this purpose, we propose an image mixing augmentation, which, when combined with other intensity manipulations (e.g., posterization, equalization, etc.) and geometric transformations (rotation, scale, and translation), can effectively generate a diverse set of out-of-distribution images. As discussed in [7], these extremely distorted images can potentially improve the performance of an SSL method. We briefly explain our image mixing methods and refer the reader to our supplementary material for the detailed composition of the $T_{e}$ module.

Cross-dataset image mixing: Our image mixing technique aims to expand the unlabeled dataset by cutting a random region from a labeled image and pasting it into an unlabeled image. The prediction for a mixed image is compared for consistency against the prediction for the labeled image only in the pasted region, and against the prediction result for the unlabeled image in all other pixels. Our image mixing method is inspired by [27,12], but differs from [27], which is data augmentation for image classification, and from [12], which mixes only same-domain images.

Exclusive transformations: Applying diverse transformations to the inputs of both student and teacher networks, however, either leads to model divergence during training or results in only a marginal improvement over a supervised baseline. We have experimentally determined that it is critical to use the diverse transformations exclusively for the inputs to the student network and apply only mild transformations to the inputs of the teacher network. Intuitively, this design makes sense because the success of the consistency loss, especially for unlabeled data, relies on a healthy reference prediction, which in our case is generated by 


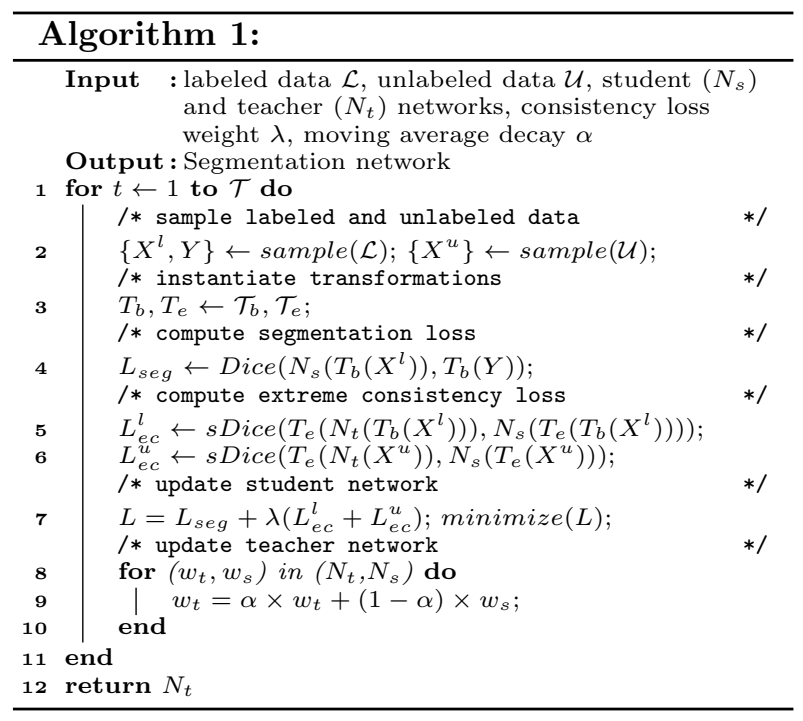

* update student networ

/* update teacher network

for $\left(w_{t}, w_{s}\right)$ in $\left(N_{t}, N_{s}\right)$ do

$w_{t}=\alpha \times w_{t}+(1-\alpha) \times w_{s}$
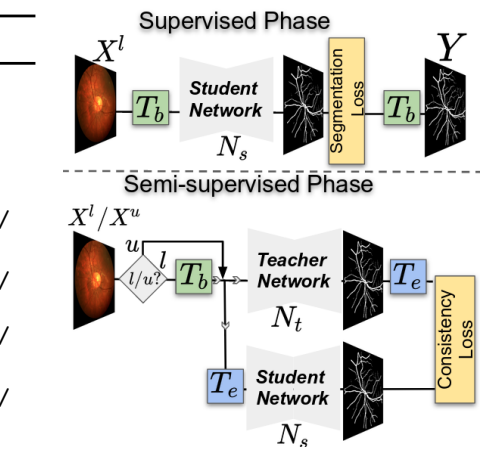

Fig. 1: (left) Algorithm 1 shows the pseudocode of our semi-supervised learning method based on extreme consistency. sDice denotes soft dice. (top right) Schematic of the supervised phase (Lines 2-4) and semi-supervised phase (Lines 5-6). (bottom right) Sigmoid ramp-up function used for consistency loss weight (Line 7).

the teacher network. It is therefore necessary to feed "easy" examples to the teacher while sending the "hard" examples to the student. Omitting this critical design choice may have shifted previous works towards consistency based on just a narrow set of transformations, leading to sub-optimal improvements.

Pseudocode: Fig. 1 presents the pseudocode of our SSL method. In each iteration, a batch of labeled $\left\{X^{l}, Y\right\}$ and unlabeled images $\left\{X^{u}\right\}$ are sampled. During the the supervised phase, the labeled batch is first sent to $T_{b}$ for mild augmentation, and then to the student network to compute the segmentation loss $L_{\text {seg }}=\operatorname{Dice}\left(N_{s}\left(T_{b}\left(X^{l}\right)\right), T_{b}(Y)\right)$. For unsupervised learning, we compute the extreme consistency losses $L_{e c}^{l}$ and $L_{e c}^{u}$ for both the labeled and unlabeled data. For the former, this is realized by first applying extreme transformations $T_{e}$ to the batch of images before feeding it to the student network $N_{s}\left(T_{e}\left(T_{b}\left(X^{l}\right)\right)\right.$. To generate the reference predictions for the consistency loss, the original labeled batch is sent to the teacher network and the equivalent extreme transformations $T_{e}\left(N_{t}\left(T_{b}\left(X^{l}\right)\right)\right)$ are applied to its predictions. The extreme consistency loss $L_{e c}^{l}$ is then computed by comparing the two outputs through a soft Dice function (both outputs are probabilistic). The extreme consistency loss for the unlabeled batch $L_{e c}^{u}$ is computed similarly, with the difference being that no basic transformation $T_{b}$ is applied to the unlabeled data. This choice is made because the student and teacher networks do not have the ground truth for unlabeled images during training; hence, applying mild transformations may degrade the quality of 
reference predictions for the unlabeled data. Once the three losses are computed, the student network is updated by minimizing $L_{s e g}+\lambda\left(L_{e c}^{l}+L^{u} l_{e c}\right)$, where $\lambda$ is governed by a sigmoid function (Fig. 1). Finally, the teacher network is updated by the moving average filter. We use the teacher network during inference.

\section{Experiments}

Datasets: We employ a skin lesion segmentation dataset (ISIC'17: 2,000/150/600) and two fundus image datasets (HRF [3]: 30/6/9 and STARE[14]: 12/4/4) in our experiments (tr/val/test). The relatively small size of fundus datasets is due to the difficulty and high cost associated with vessel annotation, which makes them a practical and meaningful choice for addressing the limited annotation problem. The selected fundus datasets also show great variability in terms of pathologies as well as variations in scanner devices. For instance, HRF consists of high-quality fundus images acquired by a modern Canon scanner whereas the STARE images are acquired by older scanners and exhibit more pathologies. Therefore, this pair of datasets is suitable for studying the domain shift problem. The skin dataset, on the other hand, is far larger than the fundus datasets, facilitating the large-scale evaluation of the suggested method. This dataset has also been used as a benchmark by one of the competing methods [18], which allows us to draw more direct comparisons with it.

Experiment protocols: For the limited annotation problem, we use all three datasets by dividing their training sets into labeled and unlabeled subsets, resulting in $\mathcal{L} / \mathcal{U}$ splits of 50/1,950 for ISIC, 3/27 for HRF, and 2/10 for STARE. For the domain shift problem, we use HRF as the source and STARE as the target dataset; that is, $\mathcal{L}$ and $\mathcal{U}$ consist of the training splits of HRF and STARE, respectively. For realistic evaluation, we choose the best segmentation model based on the validation split of the source dataset, and then evaluate the best model on the test split of the target dataset. Similar data splits and evaluation strategies have been adopted in previous works.

Competing baselines: For the limited annotation problem, we compare our method with two SSL methods, [6] and [18], which use consistency loss based on noise perturbations and affine transformations, respectively. The primary baselines for the domain shift problem are unsupervised domain adaptation methods suggested in [8] and [21]. The secondary baselines are the above SSL methods ([6] and [18]), which can potentially be used for domain adaptation as well.

Implementation details: For a fair comparison, as suggested in [20], we have implemented our method as well as all competing baselines in the same codebase. Thus, all methods benefit from the same data augmentation and further operate on the same segmentation network (exception being [21] requiring GroupNorm [25]). We have also optimized the weight function of the consistency loss for each method and dataset, by evaluating different configurations on the validation split. See our supplementary material for the best hyper-parameters. 
Table 1: 10-trial comparison between our method and the competing baselines for (a) limited annotation and (b) the domain shift problem. In both tables, the goal is to bridge the performance gap between the lower bound (LB) and upper bound (UB) performance levels. To mimic limited annotation, we have divided the training split $D_{t r}$ of each of the datasets into a small labeled subset $\mathcal{L}$ and a large subset $\mathcal{U}$ whose annotations are removed. As described in the text, the sizes of $D_{t r}, \mathcal{L}, \mathcal{U}$ differ from one dataset to another. For domain adaptation, we use HRF as the source and STARE as the target dataset. We use the training split of STARE after removing the labels (unsupervised), denoted as $\not D_{t r}^{S}$. $p$-values compare our method with the second best.

(a) Limited annotation

\begin{tabular}{lcccc}
\hline \multicolumn{1}{c}{ Method } & \multicolumn{4}{c}{ Dice: $\mu(\sigma)$} \\
\hline & Tr set & STARE & HRF & ISIC'17 \\
\hline LB (U-Net) & $\mathcal{L}$ & $0.806(0.003)$ & $0.807(0.012)$ & $0.775(0.005)$ \\
LB (UNet++) & $\mathcal{L}$ & $0.797(0.007)$ & $0.812(0.003)$ & $0.781(0.004)$ \\
Li et al. [18] & $\mathcal{L}+\mathcal{U}$ & $0.809(0.006)$ & $0.811(0.006)$ & $\mathbf{0 . 7 9 7}(\mathbf{0 . 0 0 5})$ \\
Cui et al. [6] & $\mathcal{L}+\mathcal{U}$ & $\mathbf{0 . 8 1 4}(\mathbf{0 . 0 1 3})$ & $\mathbf{0 . 8 1 3 ( 0 . 0 0 5 )}$ & $0.774(0.016)$ \\
Proposed & $\mathcal{L}+\mathcal{U}$ & $\mathbf{0 . 8 2 4}(\mathbf{0 . 0 0 7})$ & $\mathbf{0 . 8 2 8}(\mathbf{0 . 0 0 2})$ & $\mathbf{0 . 8 0 6}(\mathbf{0 . 0 0 5})$ \\
\hline UB (U-Net) & $D_{t r}$ & $0.836(0.006)$ & $0.838(0.001)$ & $0.840(0.001)$ \\
UB (UNet++) & $D_{t r}$ & $0.827(0.005)$ & $0.839(0.003)$ & $0.832(0.002)$ \\
\hline$p$-value & \multicolumn{5}{|c}{$p<0.001$} & $p<0.05$ \\
\hline
\end{tabular}

\begin{tabular}{|c|c|c|c|}
\hline Method & Tr set & Val set Test Set & Dice: $\mu(\sigma)$ \\
\hline LB (U-Net) & $D_{t r}^{H}$ & $D_{\text {test }}^{S}$ & $0.678(0.010)$ \\
\hline LB (UNet ++ ) & $D_{t r}^{H}$ & $D_{\text {test }}^{S}$ & $0.655(0.014)$ \\
\hline Li et al. [18] & $D_{t r}^{H}+D_{t r}^{S}$ & $D_{\text {test }}^{S}$ & $0.712(0.005)$ \\
\hline Cui et al. [6] & $D_{t r}^{H}+D_{t r}^{S}$ & $D_{\text {test }}^{S}$ & $0.704(0.012)$ \\
\hline Dong et al. [8] & $D_{t r}^{H}+D_{t r}^{S}$ & $D_{\text {test }}^{S}$ & $0.668(0.017)$ \\
\hline Perone et al. [21] & $D_{t r}^{H}+D_{t r}^{S}$ & $D_{\text {test }}^{S}$ & $0.723(0.012)$ \\
\hline Proposed & $D_{t r}^{H}+D_{t r}^{S}$ & $D_{\text {test }}^{S}$ & $0.736(0.004)$ \\
\hline $\mathrm{UB}(\mathrm{U}-\mathrm{Ne}$ & $D_{t r}^{S}$ & $D_{\text {test }}^{S}$ & $0.833(0.005)$ \\
\hline UB (UNet++) & $D_{t r}^{S}$ & $D_{\text {test }}^{S}$ & $0.827(0.005)$ \\
\hline$p$-value & & & $p<0.05$ \\
\hline
\end{tabular}

Segmentation Architecture: We use a U-Net with 4 blocks in the encoder and decoder. Each block consists of 2 Conv-Bn-Relu followed by a 2x2 max-pooling layer in the encoder and an up-sampling layer based on bi-linear interpolation in the decoder. To avoid overfitting, we place a dropout layer with $50 \%$ drop rate right after each encoder block. We use the Dice loss during training. Being optimized on the validation set, this U-Net performs at par with the sophisticated, heavy-weight UNet++ [29] (Table 1). This observation is in line with similar findings in [16]. We thus use the U-Net for the remaining experiments.

Results for limited annotation: Table 1(a) summarizes the results. Each model is trained and evaluated in 10 trials. According to our statistical analyses, our method has significantly outperformed the best-performing SSL method for HRF and ISIC'17. Notably, our results for [18] show a $\sim 2$-point increase over the lower bound U-Net in the ISIC'17 dataset, which is consistent with the original publication where the same dataset is used for evaluation. Our method has further outperformed the second-best method in STARE in both the mean and standard deviation.

Results for domain shift: The results are summarized in Table 1(b). Each model is trained and evaluated in 10 trials. Our method achieves a $\sim 6$-point increase in Dice over the lower bound performance (U-Net), on average $\sim 3$-point increase over the SSL methods, and a 1-point increase over the best performing domain adaptation method. All improvements are statistically significant. These results demonstrate the ability of our model to handle domain shifts.

Ablation study: Table 2 summarizes the results of our ablation study conducted on the HRF dataset. First, we studied the necessity of diverse transformations by using one transformation type at a time, observing significant drops in Dice 
Table 2: 10-trial ablation study for HRF. We have split the results into three tables: (a) the necessity of diverse transformations and our image mixing method (last two rows); (b) impact of exclusive transformations (+Exclusive), use of unlabeled data $(+\mathcal{U})$, and the necessity of a mean teacher network (+Teacher); (c) whether a supervised model with extreme augmentation is as effective as our method.

\begin{tabular}{ccccc}
\multicolumn{5}{l}{ (a) Diverse transformations } \\
\hline +Inten. & Geo. + & Mixing & Dice: $\mu(\sigma)$ & $\Delta$ Dice $(\%)$ \\
\hline$\checkmark$ & $\mathbf{X}$ & $\mathbf{X}$ & $0.817(0.002)$ & $-1.3 \%$ \\
$\mathbf{X}$ & $\checkmark$ & $\mathbf{X}$ & $0.814(0.020)$ & $-1.6 \%$ \\
$\mathbf{X}$ & $\mathbf{X}$ & $\checkmark$ & $0.817(0.008)$ & $-1.3 \%$ \\
$\checkmark$ & $\checkmark$ & $\mathbf{X}$ & $0.818(0.019)$ & $-1.3 \%$ \\
$\checkmark$ & $\checkmark$ & $\checkmark$ & $\mathbf{0 . 8 2 8 ( 0 . 0 0 2 )}$ & $0.0 \%$ \\
\hline
\end{tabular}

\begin{tabular}{|c|c|c|c|c|}
\hline +Exclusive & $+\mathcal{U}$ & +Teacher & Dice: $\mu(\sigma)$ & $\Delta$ Dice $(\%)$ \\
\hline$x$ & $\checkmark$ & $\checkmark$ & $0.803(0.005)$ & $-3.0 \%$ \\
\hline$\checkmark$ & $x$ & $\checkmark$ & $0.816(0.002)$ & $-1.4 \%$ \\
\hline$\checkmark$ & $\checkmark$ & $x$ & $0.823(0.004)$ & $-0.6 \%$ \\
\hline$\checkmark$ & $\checkmark$ & $\checkmark$ & $0.828(0.002)$ & $0.0 \%$ \\
\hline \multicolumn{5}{|c|}{ (c) Supervised model with extreme augmentation } \\
\hline \multicolumn{2}{|c|}{ model } & $T_{b}$ & $T_{e}$ & Dice \\
\hline \multicolumn{2}{|l|}{ Sup. U-Net } & $\checkmark$ & & $0.807(0.012)$ \\
\hline \multicolumn{2}{|l|}{ Sup. U-Net } & $\checkmark$ & $\checkmark$ & $0.801(0.007)$ \\
\hline \multicolumn{2}{|c|}{ Ours: SSL U-Net } & $\checkmark$ & $\checkmark$ & $0.828(0.002)$ \\
\hline
\end{tabular}

$(p<0.05)$ compared to using diverse transformations. We further compared diverse transformation with and without our image mixing method (the last two rows in Table 2(a)), observing a substantial decrease in average Dice without image mixing. It is also clear that image mixing significantly reduces the standard deviation of Dice, which translates to far more stable training sessions and reproducible models. Second, we removed the exclusion principle, applying the diverse transformations to the inputs of both student and teacher networks. This change led to a significant drop in Dice (3\%), degrading the performance to that of the lower bound supervised model, motivating the the need for the exclusive transformations. Third, we excluded unlabeled data, computing segmentation and consistency losses using only labeled data. This configuration led to $1.4 \%$ drop in Dice. Fourth, we studied the effect of replacing the teacher with the student network, which is known as the $\Pi$ model [17], observing a $0.6 \%$ drop in Dice. Fifth, we trained the lower bound U-Net with extreme data augmentation $\left(T_{e}\right)$, observing no significant gains, which rules out the possibility that the gains by our SSL method are due to extensive data augmentation. These experiments underline the importance of each individual component of our method.

\section{Conclusion}

We presented Extreme Consistency as a simple yet effective semi-supervised learning framework. Although our method is intuitive and simple to implement, it demonstrates a substantial performance gain over several baselines across multiple datasets in handling both limited annotation and domain shift problems. Through extensive ablation studies, we further demonstrated that each component of our method contributes to its success. While our empirical results are strong, surpassing state-of-the-art performances in most of the applications, important future work will include the exploration of applications of extreme consistency beyond image segmentation and the currently used datasets. 


\section{References}

1. Baur, C., Albarqouni, S., Navab, N.: Semi-supervised deep learning for fully convolutional networks. In: International Conference on Medical Image Computing and Computer-Assisted Intervention. pp. 311-319. Springer (2017)

2. Bortsova, G., Dubost, F., Hogeweg, L., Katramados, I., de Bruijne, M.: Semisupervised medical image segmentation via learning consistency under transformations. In: International Conference on Medical Image Computing and ComputerAssisted Intervention. pp. 810-818. Springer (2019)

3. Budai, A., Bock, R., Maier, A., Hornegger, J., Michelson, G.: Robust vessel segmentation in fundus images. International journal of biomedical imaging $\mathbf{2 0 1 3}$ (2013)

4. Chen, C., Dou, Q., Chen, H., Heng, P.A.: Semantic-aware generative adversarial nets for unsupervised domain adaptation in chest x-ray segmentation. In: International Workshop on Machine Learning in Medical Imaging. pp. 143-151. Springer (2018)

5. Chen, C., Dou, Q., Chen, H., Qin, J., Heng, P.A.: Synergistic image and feature adaptation: Towards cross-modality domain adaptation for medical image segmentation. In: Proceedings of the AAAI Conference on Artificial Intelligence. vol. 33, pp. 865-872 (2019)

6. Cui, W., Liu, Y., Li, Y., Guo, M., Li, Y., Li, X., Wang, T., Zeng, X., Ye, C.: Semi-supervised brain lesion segmentation with an adapted mean teacher model. In: International Conference on Information Processing in Medical Imaging. pp. 554-565. Springer (2019)

7. Dai, Z., Yang, Z., Yang, F., Cohen, W.W., Salakhutdinov, R.R.: Good semisupervised learning that requires a bad gan. In: Advances in neural information processing systems. pp. 6510-6520 (2017)

8. Dong, N., Kampffmeyer, M., Liang, X., Wang, Z., Dai, W., Xing, E.: Unsupervised domain adaptation for automatic estimation of cardiothoracic ratio. In: International conference on medical image computing and computer-assisted intervention. pp. 544-552. Springer (2018)

9. Duan, K., Bai, S., Xie, L., Qi, H., Huang, Q., Tian, Q.: Centernet: Object detection with keypoint triplets. arXiv preprint arXiv:1904.08189 (2019)

10. E. Riba, D. Mishkin, D.P.E.R., Bradski, G.: Kornia: an open source differentiable computer vision library for pytorch. In: Winter Conference on Applications of Computer Vision (2020), https://arxiv.org/pdf/1910.02190.pdf

11. E. Riba, M. Fathollahi, W.C.E.R., Bradski, G.: torchgeometry: when pytorch meets geometry (2018), https://drive.google.com/file/d/1xiao1Xj9WzjJ08YY_ nYwsthE-wxfyfhG/view? usp=sharing

12. French, G., Aila, T., Laine, S., Mackiewicz, M., Finlayson, G.: Consistency regularization and cutmix for semi-supervised semantic segmentation. arXiv preprint arXiv:1906.01916 (2019)

13. Giger, M.L.: Whole Brain Segmentation and Labeling from CT Using Synthetic MR Images. Journal of the American College of Radiology 15(3), 512-520 (2018). https://doi.org/10.1016/j.jacr.2017.12.028

14. Hoover, A., Kouznetsova, V., Goldbaum, M.: Locating blood vessels in retinal images by piecewise threshold probing of a matched filter response. IEEE Transactions on Medical imaging 19(3), 203-210 (2000)

15. Huo, Y., Xu, Z., Moon, H., Bao, S., Assad, A., Moyo, T.K., Savona, M.R., Abramson, R.G., Landman, B.A.: Synseg-net: Synthetic segmentation without target modality ground truth. IEEE transactions on medical imaging 38(4), 1016-1025 (2018) 
16. Isensee, F., Kickingereder, P., Wick, W., Bendszus, M., Maier-Hein, K.H.: No new-net. In: International MICCAI Brainlesion Workshop. pp. 234-244. Springer (2018)

17. Laine, S., Aila, T.: Temporal ensembling for semi-supervised learning. arXiv preprint arXiv:1610.02242 (2016)

18. Li, X., Yu, L., Chen, H., Fu, C.W., Heng, P.A.: Transformation consistent selfensembling model for semi-supervised medical image segmentation. arXiv preprint arXiv:1903.00348 (2019)

19. Mondal, A.K., Dolz, J., Desrosiers, C.: Few-shot 3d multi-modal medical image segmentation using generative adversarial learning. arXiv preprint arXiv:1810.12241 (2018)

20. Oliver, A., Odena, A., Raffel, C.A., Cubuk, E.D., Goodfellow, I.: Realistic evaluation of deep semi-supervised learning algorithms. In: Advances in Neural Information Processing Systems. pp. 3235-3246 (2018)

21. Perone, C.S., Ballester, P., Barros, R.C., Cohen-Adad, J.: Unsupervised domain adaptation for medical imaging segmentation with self-ensembling. NeuroImage 194, 1-11 (2019)

22. Sedai, S., Mahapatra, D., Hewavitharanage, S., Maetschke, S., Garnavi, R.: Semisupervised segmentation of optic cup in retinal fundus images using variational autoencoder. In: International Conference on Medical Image Computing and Computer-Assisted Intervention. pp. 75-82. Springer (2017)

23. Sohn, K., Berthelot, D., Li, C.L., Zhang, Z., Carlini, N., Cubuk, E.D., Kurakin, A., Zhang, H., Raffel, C.: Fixmatch: Simplifying semi-supervised learning with consistency and confidence. arXiv preprint arXiv:2001.07685 (2020)

24. Tajbakhsh, N., Jeyaseelan, L., Li, Q., Chiang, J., Wu, Z., Ding, X.: Embracing imperfect datasets: A review of deep learning solutions for medical image segmentation. arXiv preprint arXiv:1908.10454 (2019)

25. Wu, Y., He, K.: Group normalization. In: Proceedings of the European Conference on Computer Vision (ECCV). pp. 3-19 (2018)

26. Yu, L., Wang, S., Li, X., Fu, C.W., Heng, P.A.: Uncertainty-aware self-ensembling model for semi-supervised $3 \mathrm{~d}$ left atrium segmentation. In: International Conference on Medical Image Computing and Computer-Assisted Intervention. pp. 605-613. Springer (2019)

27. Yun, S., Han, D., Oh, S.J., Chun, S., Choe, J., Yoo, Y.: Cutmix: Regularization strategy to train strong classifiers with localizable features. In: Proceedings of the IEEE International Conference on Computer Vision. pp. 6023-6032 (2019)

28. Zhang, Y., Yang, L., Chen, J., Fredericksen, M., Hughes, D.P., Chen, D.Z.: Deep adversarial networks for biomedical image segmentation utilizing unannotated images. In: International Conference on Medical Image Computing and ComputerAssisted Intervention. pp. 408-416. Springer (2017)

29. Zhou, Z., Siddiquee, M.M.R., Tajbakhsh, N., Liang, J.: Unet++: A nested u-net architecture for medical image segmentation. In: Deep Learning in Medical Image Analysis and Multimodal Learning for Clinical Decision Support, pp. 3-11. Springer (2018) 


\section{Supplementary Material}

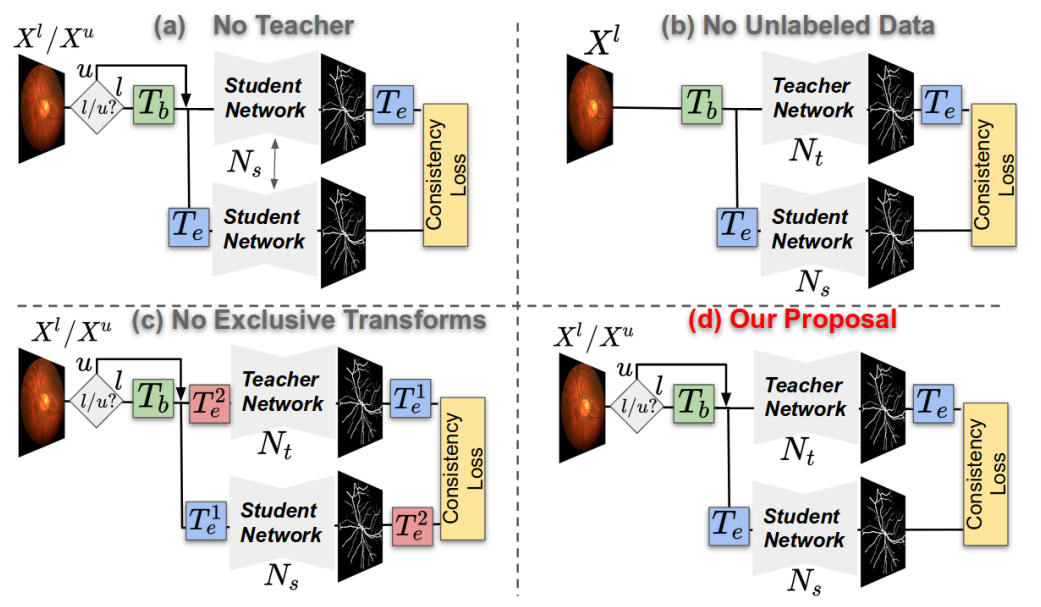

Fig. 1: Different variants of our method used in the ablation study. The supervised phase is the same for all variants; hence, not shown here. Transformation blocks with the same color perform the same transformation on the input data. (a) the teacher network is replaced with the student network. (b) consistency loss is computed for only labeled data. (c) Diverse transformation are also applied to the teacher. (d) The proposed architecture, which uses teacher, unlabeled data, and exclusive perturbations.
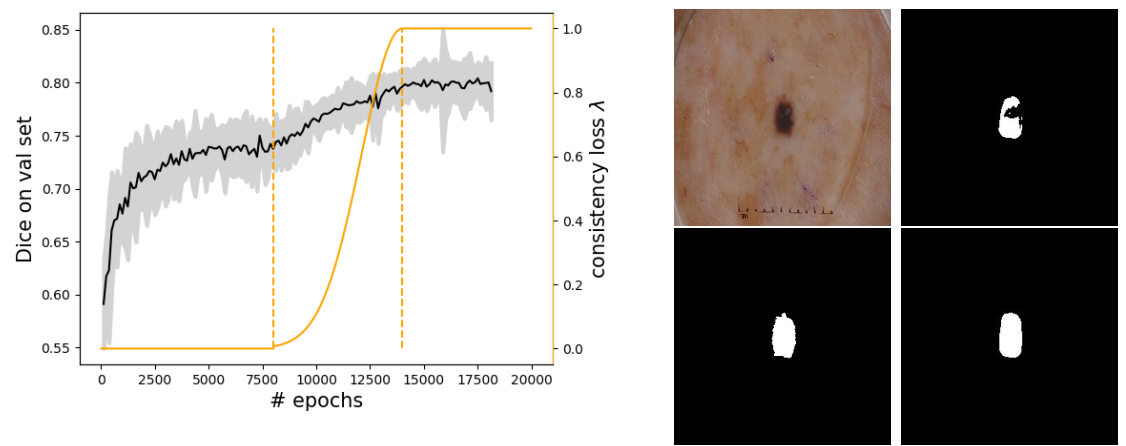

Fig. 2: (left) Effect of extreme consistency (EC) loss on the convergence of the teacher network. ISIC' 17 is used for training and evaluation. The shaded plot indicates the $95 \%$ confidence interval of 10 trials. The initiation of EC at 8,000 epochs starts a new increasing trend in the validation Dice, which results in a marked improvement over the baseline supervised model. (right) clockwise: original image, segmentation without EC, segmentation with EC, and ground truth (GT). 
Table 1: Hyper-parameters for our method and other consistency-based methods used for comparison. For each method and dataset, hyper-parameters are tuned on the corresponding validation set. We use a moving average decay of $\alpha=0.999$ and further employ the Adam optimizer with a learning rate of 0.001 .

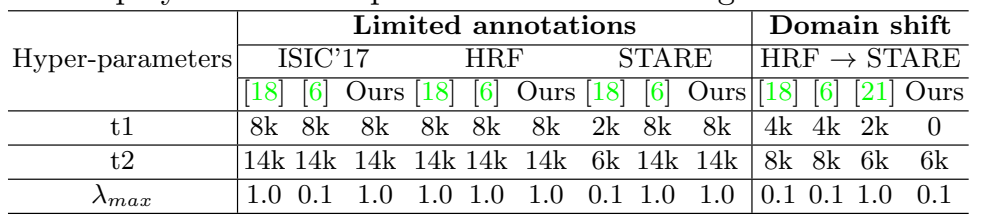

Table 2: List of basic and extreme augmentations along with their corresponding parameters. Extreme augmentation consists of a random selection of 1 geometric and 3 intensity transformations followed by a $50 \%$ chance of applying image mixing. We use kornia $[10,11]$ to implement some of the transformations below.

\begin{tabular}{|c|c|c|c|}
\hline Augmentation & Description & Parameter & Values \\
\hline \multicolumn{4}{|c|}{ BASIC AUGMENTATIONS } \\
\hline Saturation Jitter & Scale the saturation channel by a random constant & Saturation factor & $\mathrm{U}(0.9,1.1)$ \\
\hline Hue Jitter & Shifts the hue channel by a random constant & Hue factor & $\mathrm{U}(-0.1,0.1)$ \\
\hline Flip & Flips the image along the vertical or horizontal axis & & \\
\hline Channel Shuffle & $\begin{array}{l}\text { Randomizes the order of image channels } \\
\text {. }\end{array}$ & & \\
\hline & & Rotation & $\mathrm{U}(-10,10)$ \\
\hline Affine & Applies affine transformation consisting of rotation, scaling and translation & Scale & $\mathrm{U}(0.75,1.25)$ \\
\hline & & Translation & $\mathrm{U}(-32,32)$ \\
\hline \multicolumn{4}{|c|}{ EXTREME AUGMENTATIONS } \\
\hline \multicolumn{4}{|l|}{ Intensity } \\
\hline Autocontrast & $\begin{array}{l}\text { Maximizes the image contrast by stretching the min and max } \\
\text { of each image channel and then blends it with the original image. }\end{array}$ & Blending ratio & $\mathrm{U}(0,1)$ \\
\hline Brightness & $\begin{array}{l}\text { Adjusts the image brightness. An enhancement factor of } 0 \\
\text { gives a black image and } 1 \text { gives the original image. }\end{array}$ & Enhancement factor & $\mathrm{U}(0.5,1)$ \\
\hline Color & $\begin{array}{l}\text { Adjusts the color balance of an image. An enhancement factor of } \\
0 \text { gives a black-and-white image while } 1 \text { gives the original image. }\end{array}$ & Enhancement factor & $\mathrm{U}(0,1)$ \\
\hline Contrast & Decreases the image contrast. An enhancement factor of & Enhancement factor & $\mathrm{U}(0.5,1)$ \\
\hline Equalize & Equalizes image channels and then blends it with the original image. & Blending ratio & $\mathrm{U}(0,1)$ \\
\hline Invert & Inverts all pixel intensities and then blends it with the original image. & Blending ratio & $\begin{array}{l}\mathrm{U}(0,0.25) \text { or } \\
\mathrm{U}(0.75,1)\end{array}$ \\
\hline Solarize & Inverts all pixels whose intensities are above a threshold & Threshold & $\mathrm{U}(0.3,1)$ \\
\hline Posterize & Reduces number of bits for each color channel & Number of bits & $\mathrm{U}(4,8)$ \\
\hline \multicolumn{4}{|l|}{ Geometric } \\
\hline Rotation & Rotates the image & Extent & $\mathrm{U}(-10,10)$ \\
\hline Translation & Translates the image horizontally or vertically & Extent & $\mathrm{U}(-32,32)$ \\
\hline Scale & Scales the image & Scale factor & $\mathrm{U}(0.75,1.25)$ \\
\hline Flip & Randomly flips along the vertical or horizontal axis & & \\
\hline \multicolumn{4}{|l|}{ Image Mixing } \\
\hline Cross-dataset image manipulation & $\begin{array}{l}\text { Mixes two images by copying a square patch from one to the other. } \\
\text { The patch location is random and size is a random fraction of the image. }\end{array}$ & Size ratio & $\mathrm{U}(0,0.5)$ \\
\hline
\end{tabular}
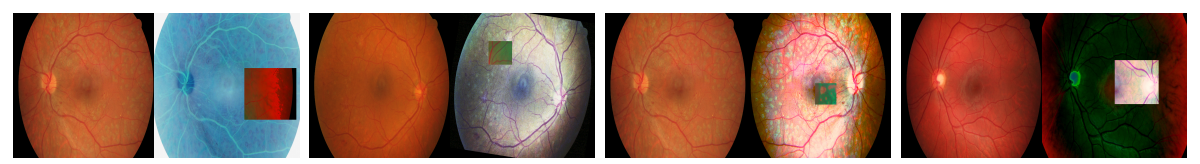

Fig. 3: Examples of original images and their extremely augmented versions, where the latter is produced using intensity, geometric, and mixing transformations. 